\title{
PROgNOSTIC FACTORS FOR MORTALITY IN STROKE PATIENTS. \\ DOES PREHOSPITAL DELAY PLAY A ROLE?
}

While mortality and dependency are well known consecuences of the delay in the start of reperfusion therapies, prehospital delay has not been stablished as a predictor for mortality in all stroke patients. We conducted a study in stroke patients to investigate the role of prehospital delay in mortality.
382 Patients with acute stroke or TIA were prospectively included. Sociodemographic factors, premorbid conditions, and clinical, biochemical and hematological parameters that were considered as possible prognostic factors in previous studies were collected, as well as data on PD and mortality. Multivariate logistic regression analyses were conducted to identify factors related to achieving functional independency three months after stroke.

\section{RESULTS}

Table 1. Sample Characteristics

\begin{tabular}{|c|c|c|c|c|}
\hline Variable & $\begin{array}{c}\text { Total } \\
(n=382)\end{array}$ & $\begin{array}{r}P D<180^{\prime} \\
(n=186)\end{array}$ & $\begin{array}{l}P D>180^{\prime} \\
(n=196)\end{array}$ & $\mathbf{p}$ \\
\hline Age (Mean;y) & 73,9 & 73,5 & 74,3 & ns \\
\hline Sex (Male;\%) & 54,2 & 52,2 & 56,1 & ns \\
\hline TIA (\%) & 19,1 & 22,0 & 16,3 & ns \\
\hline $\begin{array}{l}\text { Etiology (\%) } \\
\text { Aterothrombotic } \\
\text { Cardioembolic } \\
\text { Lacunar } \\
\text { Other } \\
\text { Undetermined } \\
\text { Haemorrhagic }\end{array}$ & $\begin{array}{c}13,4 \\
24,9 \\
23,3 \\
6,5 \\
20,7 \\
11,3\end{array}$ & $\begin{array}{c}12,4 \\
30,6 \\
17,7 \\
9,1 \\
17,2 \\
12,9\end{array}$ & $\begin{array}{c}14,3 \\
19,4 \\
28,6 \\
4,1 \\
24,0 \\
9,7\end{array}$ & 0,001 \\
\hline $\begin{array}{l}\text { OCSP (\%) } \\
\text { TACI }\end{array}$ & 13,9 & 21,61 & 6,8 & $<0,001$ \\
\hline Previous $\mathrm{mRS} \leq 2$ & 79,3 & 80,7 & 78,1 & ns \\
\hline NIHSS (Mean) & 5,64 & 7,78 & 3,61 & ns \\
\hline Hypertension (\%) & 74,6 & 76,9 & 72,5 & ns \\
\hline Diabetes Mellitus (\%) & 36,1 & 36,6 & 35,7 & ns \\
\hline Dyslipidemia (\%) & 42,9 & 44,1 & 41,8 & ns \\
\hline Atrial Fibrilation (\%) & 15,7 & 20,4 & 11,2 & 0,013 \\
\hline Ischemic Heart Disease (\%) & 15,2 & 16,7 & 13,8 & ns \\
\hline Hemoglobin (Mean;g/dL) & 13,8 & 13,9 & 13,8 & ns \\
\hline Albumin (Mean;mg/dL) & 3,9 & 3,9 & 3,9 & ns \\
\hline
\end{tabular}

Table 2. Prognostic factors for independency 1 year after stroke

\begin{tabular}{|cccc|}
\hline Variable & OR & IC 95\% & P \\
\hline Infectious Comorbidities & 12.258 & $4.832-31.093$ & $<0,001$ \\
\hline Renal Failure & 9.053 & $2.796-29,312$ & $<0,001$ \\
\hline SBP > 185mmHg & 5.665 & $1.690-18.985$ & 0,005 \\
\hline Active Smoking & 4.335 & $1.045-17.992$ & 0.043 \\
\hline Previous mRS & 1,659 & $1.209-2.277$ & 0.002 \\
\hline Onset NIHSS & 1.251 & $1.161-1.347$ & $<0,001$ \\
\hline PD $\leq 180$ & 0,136 & $0.043-0.431$ & 0.001 \\
\hline
\end{tabular}

Figure 1. Prehospital delay and mortality

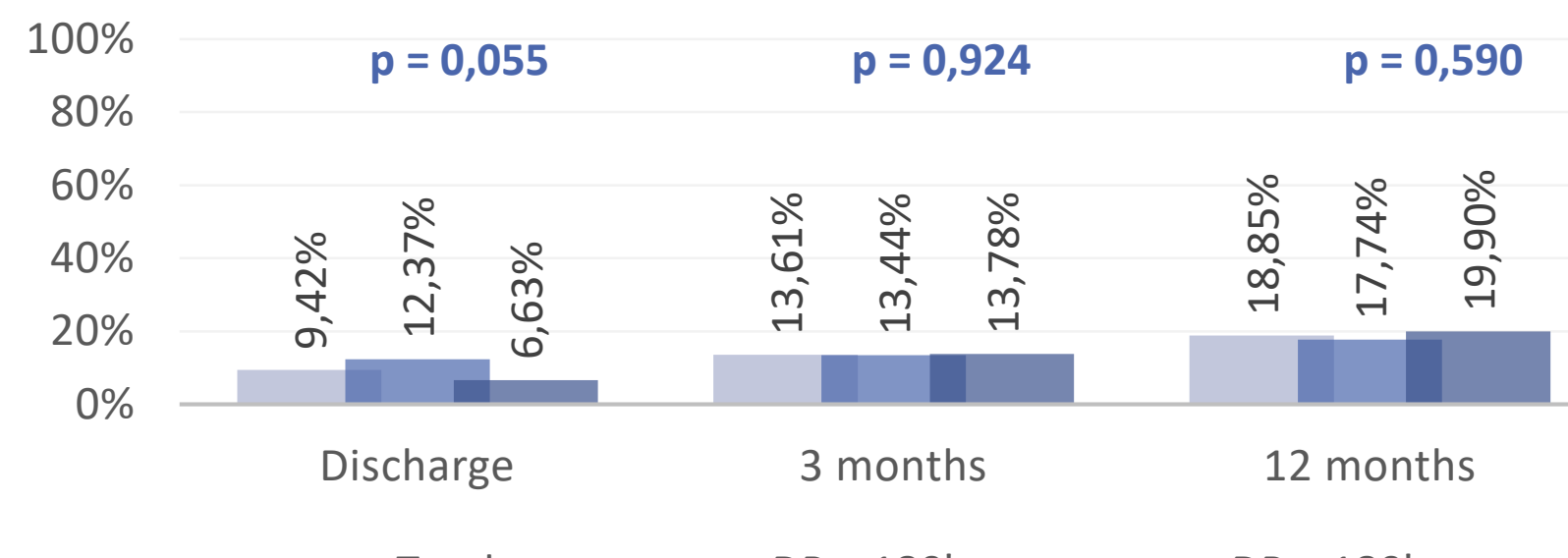

Figure 2. Onset mRS

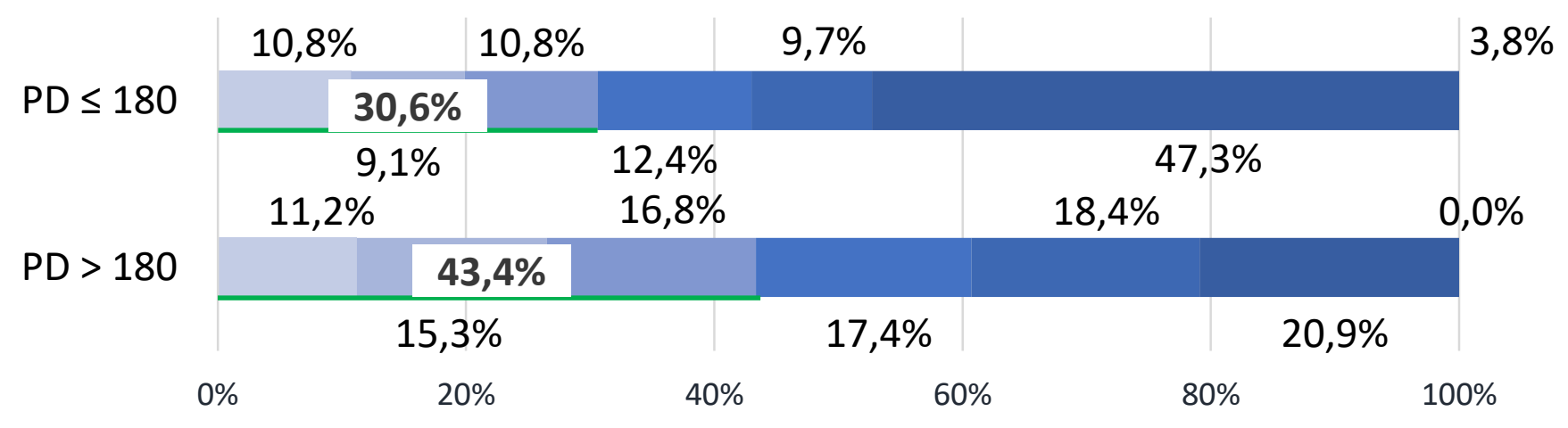

Figure 3. $\mathrm{mRS}$ at discharge

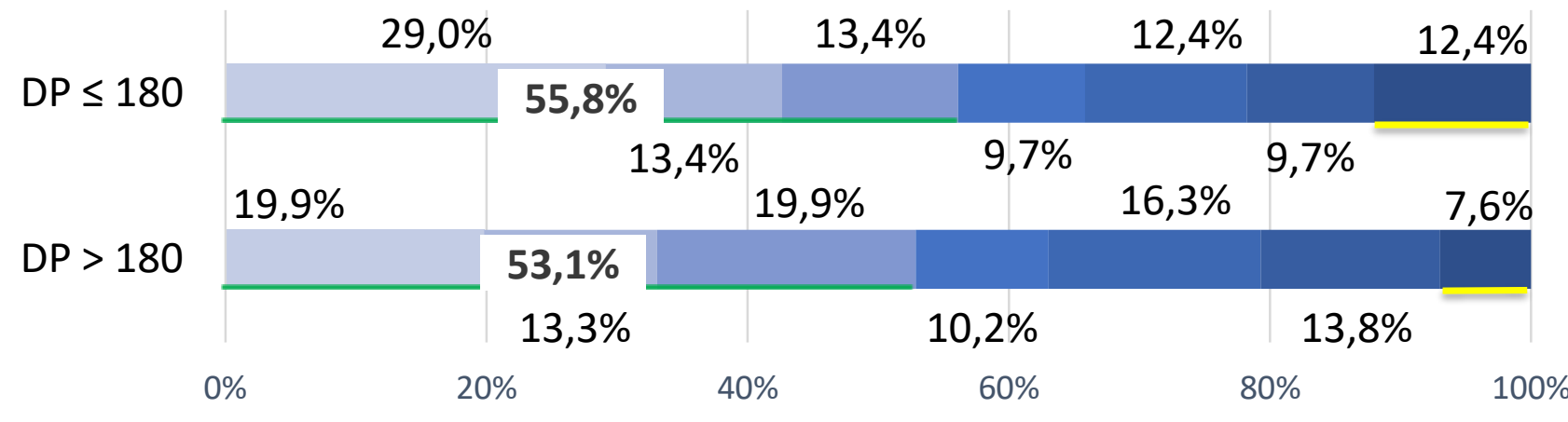

Figure 4. mRS 3 months after onset

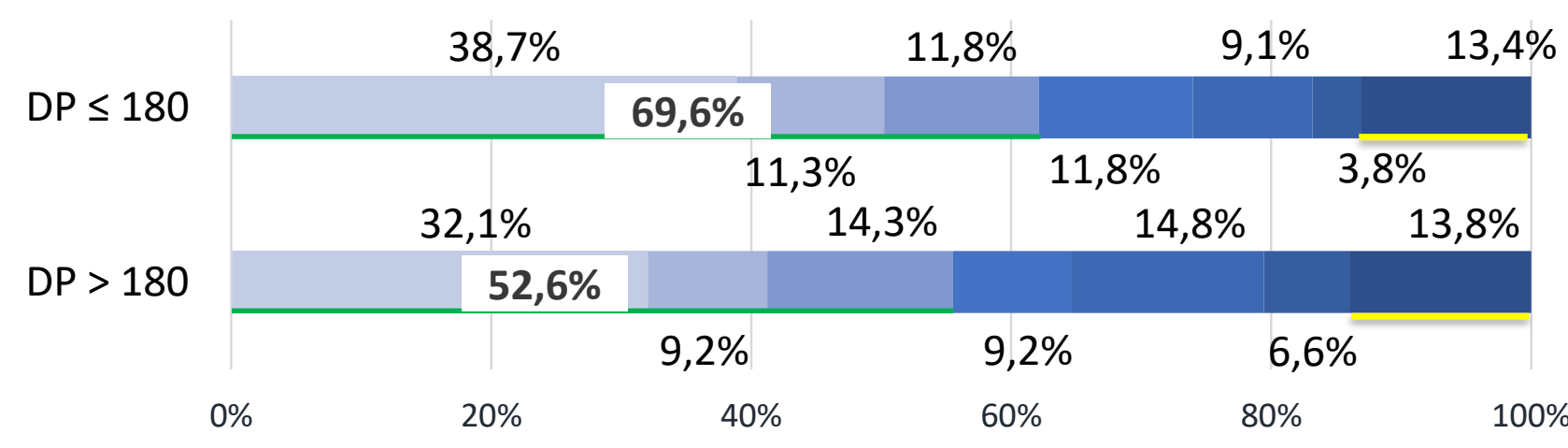

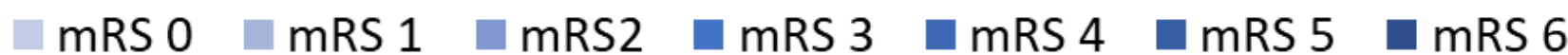

\section{CONCLUSIONS}

A prehospital delay of less than three hours reduces mortality three months after stroke onset. Future interventions should be directed towards increasing the percentage of patients arriving the hospital in the first three hours, which would not only increase thrombolysis and thrombectomy rates, but also decrease mortality.

\section{BIBLIOGRAPHY}

Hacke W, Donnan G, Fieschi C, Kaste M, von Kummer R, Broderick JP, et al. Association of outcome with early stroke treatment: pooled analysis of ATLANTIS, ECASS, and NINDS rt-PA stroke trials. Lancet. 2004;363(9411):768-74. 2. Davalos A, Castillo J, Martinez-Vila E. Delay in neurological attention and stroke outcome. Cerebrovascular Diseases Study Group of the Spanish Society of Neurology. Stroke. 1995;26(12):2233-7. Kimura Y, Yamada M, Itagaki A, 3. Qureshi Al, Kirmani JF, Sayed MA, Safdar A, Ahmed S, Ferguson R, et al. Time to hospital arrival, use of thrombolytics, and in-hospital outcomes in ischemic stroke. Neurology. 2005;64(12):2115-20.

4. Naganuma M, Toyoda K, Nonogi H, Yokota C, Koga M, Yokoyama H, et al. Early hospital arrival improves outcome at discharge in ischemic but not hemorrhagic stroke: A prospective multicenter study. Cerebrovasc Dis. 2009;28(1):33-8. 5. Denti L, Artoni A, Scoditti U, Gatti E, Bussolati C, Ceda GP. Pre-hospital Delay as Determinant of Ischemic Stroke Outcome in an Italian Cohort of Patients Not Receiving Thrombolysis. J Stroke Cerebrovasc Dis. 2016;25(6):1458-66. 